\title{
Coordinating supplier-retailer using multiple common replenishment epochs with retailers' choices
}

\author{
Juhwen Hwang, Su-Hwa Wu* \\ National Dong Hwa University (Taiwan, R.O.C.) \\ him@mail.ndhu.edu.tw, marina@mail.ndhu.edu.tw \\ * Corresponding author
}

\section{Abstrad:}

Purpose Provide a coordination strategy using multiple common replenishment epochs (MCRE) for a single-supplier multi-retailer supply chain.

Design/mathoddogy/approadr. The demand of a product occurs only with a group of heterogeneous and independent retailers with constant rates, whereas all their order requests are fulfilled by the supplier. The supplier decides a set of MCREs with general price and extra bonus to entice the retailers to join any one of the MCREs, or to let them remain with their original order time epochs. A retailer is willing to participate in a CRE as long as the retailer's cost increase is within its tolerance. This paper provides a mixed integer programming to determine the MCRE strategies in order to minimize the total costs of the supplier.

Findings: The results illustrate that MCRE model provided in the paper can generate a better replenishment coordination scheme than single CRE models.

Practical implications Replenishment coordination is one of the most important mechanisms to improve the efficiency in supply chains, e.g., chain convenience stores in the modern retail industry.

Originality/value This is a follow-up research on Joint Economic Lot Size (JELS) models with a focus on multiple retailers with their replenishment coordination. 
Keywords: supply chain, inventory management, common replenishment epoch

\section{Introduction}

To obtain competitive advantages, supply chain partners often seek coordination to improve performance of the supply chain. As one of the most important supply chains in the modern retail industry, chain convenience stores have devoted significant efforts to improve supply coordination. As of 2012, the three famous convenience store chains, 7-Eleven, FamilyMart, and Couche-Tard, operate around 43,000, 17,560 and 6,000 convenience stores worldwide respectively (7-Eleven, 2012; FamilyMart, 2012; Alimentation Couche-Tard, 2012). The huge and continuously growing number of stores in chain convenience stores creates a persistent drive to improve their supply coordination. Often, a better coordination in the supply chain supports the products with an enhanced competitive advantage. In practice, the retailing industry with multiple stores would divide the distribution channels into various modules based on geographic areas, replenishment epochs, or some other managerial considerations to enhance logistic efficiency.

This paper studies a single-supplier, multiple-retailer supply chain for a single commodity. The demand of the commodity occurs only at the retailers with constant rates, and the supplier is responsible for replenishing all the retailers' requests to satisfy their demands. The supplier is the leader of the supply chain, and it provides a sequence of prescheduled epochs for all the retailers to choose their order replenishment timings. The cost disadvantages of the retailers for joining the replenishment timings shall be compensated by the supplier through quantity discounts. In addition, the costs considered in the supply chain include setup and delivery costs of the supplier, as well as holding and ordering setup costs of the retailers. The objective of the supply chain is to minimize the total cost of the supplier.

\section{Literature review}

The importance of coordination in the supply chain is evidenced by the ample growth in the coordination literature. Fugate, Sahin, and Mentzer (2006) summarize the benefits of coordination including cost reduction, risk reduction, profit increase and competitive advantages enhancement. Interested readers can refer to Goyal and Gupta (1989), Sarmah Acharya and Goyal (2006, 2008), Arshinder, Kandaa and Deshmukh (2008), Chan and Chan (2010), and Chan (2011) for the review of the buyer-vendor coordination models.

Replenishment timing is one of the essential issues in inventory management. Heterogeneous retailers pursuing their own optimizations may order at various times. Nevertheless, the variations on retailers' replenishment timings significantly increase the cost of the supplier for handling these replenishment orders. If replenishment orders can be synchronized, the relevant inventory costs can be reduced. Hence, replenishment coordination is one of the areas that research focuses on supply chain coordination, e.g., Yao and Chiou (2004), Chen and 
Chen (2005), Wu and Hwang (2011). In order to provide incentives for coordination, mechanisms such as quantity discount, buy-back/return policies, and quantity flexibility are often used (Tsay, Nahmias \& Agrawal, 1999; Sarmah et al., 2006). However, quantity discount is considered to be one of the most popular coordination mechanisms (Weng, 1995; Jorgensen and Zaccour, 2003; Sarmah et al., 2006; Li \& Wang, 2007).

Assume the supplier has all the relevant information about the retailer's replenishment information, Viswanathan and Piplani (2001) first propose a CRE (Common Replenishment Epoch) coordination model wherein the supplier offers a price discount as the compensation and incentive for all buyers to participate in the coordination scheme. Under a CRE scheme, all participating retailers release requests only at the supplier's required CREs. Their model is modified by Mishra (2004) with a selective discount policy in which some buyers are excluded under the chosen CRE and discount. Mishra solves a two CRE problem with an algorithm by segmenting buyers based on selective price discounts prior to assigning buyers into CREs. Piplani and Viswanathan (2004) focus on saving joint order processing costs for the orders placed by either one or both CREs at the same time. Feng and Viswanathan (2007) further reveal the effectiveness of coordinating supplier and retailers through a single CRE facing uncertain demand. Yao and Chiou (2009) propose a cooperative model with a saving-sharing mechanism to further enhance the performance of the supply chain within a single CRE consideration.

Note that Viswanathan and Piplani discuss a single CRE model, and Mishra studies a selective discount model with a single CRE or two CREs. The work of Piplani and Viswanathan assumes a dependent relationship between two CREs which seems restrictive in a practical sense. Our paper adopts the framework of Viswanathan and Piplani (2001) and Mishra (2004) and extends the model into a multiple CRE (MCRE) setting in which the multiple CREs are not dependent. The implementation of a multiple CRE (MCRE) schemes of the supplier in this paper is based on the frameworks of Viswanathan and Piplani (2001) and Mishra (2004). Nevertheless, our research develops a general discount (GD) model such that the choice of MCRE to the retailers is simply based on the criterion of minimizing the supplier's total cost without pre-segmenting buyers into either one of MCREs or none.

\section{Model formulation and analysis}

Now, we are in a position to analyze a single-supplier multi-retailer replenishment problem for a retailing supply chain. In this section, we will first describe and analyze the problem of this paper. In the subsequent subsections, the problem will be formulated into two MCRE models, General Discount (GD) and Exact General Discount (EGD) MCRE. We name Mishra's model as a Selective Discount (SD) model. GD is defined as a more general model than SD model. The SD will finally select a discount of a CRE based on the cost disadvantage of a retailer, and then any retailers have to join the CRE if and only if the following condition is true: a retailer's 
disadvantage of joining the CRE is able to compensate through the discount selected. This condition is relaxed in the GD model such that every retailer is free to join a CRE as long as the minimum total cost of the supplier can be reached. The second MCRE model defined in this paper, EGD, is designed to remove redundancy of the joint order processing cost in the GD model, cf. Piplani and Viswanathan (2004), with a broader definition.

\subsection{Model description}

Consider a supply chain in which a supplier sells a product with a unit price $P$ to $m$ heterogeneous and self-governing retailers, where retailer $i=1, \ldots, m$ has constant demand rate $\lambda_{i}$, holding cost rate $h_{i}$, and order setup cost $K_{i}$. In the system, the supplier is the leader and considers an MCRE scheme with all the preferable CREs in a set $X$. The MCRE scheme is restricted to offer only $w$ CREs from $X$ for all the retailers to join. Thus, $X_{w}$ is defined as a subset of $X$ with $w$ elements numbered as CRE $j=1, \ldots, w$. Each CRE $j$ can be specified by the time interval between two consecutive ordering occasions $\tau_{j}$, i.e., $X_{w}=\left\{\tau_{1}, \ldots, \tau_{w}\right\}$.

As long as a retailer participates in a CRE $j$ scheme, the supplier offers incentives, including a selling price discount $d_{j}$ to compensate the retailer's inventory cost increase, plus a bonus $100 S_{j}$ measured as a fraction of the retailer's original inventory cost. The price discount and the bonus only pertain to the retailers selecting the CRE, regardless of any particular retailer to avoid price discrimination. The incentives offered by the supplier represent how much it is willing to pay in order to achieve a better operational efficiency. At certain point of time, the supplier processes an order from a retailer $i$ and incurs an order processing cost of $U_{i}$ if the retailer $i$ does not participate in any CRE. Conversely, the supplier incurs a joint order processing cost of $A_{S}$ for the entire MCRE orders with an additional cost of $A_{j}$ dedicated for all the orders of a CRE $j$. In addition, the supplier incurs a delivery cost of $D_{i}$ for every order placed by the retailer $i$. Under the MCRE scheme, a retailer is willing to select one of the MCREs only if its inventory cost increase is below its limit of tolerance; otherwise, the retailer will choose to stick with its original time epoch. The magnitudes of cost increase indicate the retailer's needs to adjust the changes. If the cost increases beyond the tolerance limit, some of the managerial impact may not be easily adjusted, e.g., limitations on storage and facilities, financial issues, human resources, and the product perishability, etc. Therefore, the retailer will simply reject the CRE offer. This is the rationale to incorporate retailers' tolerance limits in the model. The limit of tolerance of a retailer $i$ is denoted as a threshold $\beta_{i}>1$, which is defined as the ratio of its inventory cost after and before participating. The threshold signifies the internal cost increase tolerance of the retailer, and is a criterion for the retailer to judge the supplier's MCRE offer. Once the retailer $i$ participates in the CRE $j$, its order interval must follow $n_{i j} \tau_{j}$, where $n_{i j}$ is a positive integer. In case that the retailer $i$ selects its own order interval rather than any CRE, a dummy CRE $w+1$ is denoted as the choice of the retailer not to join the entire CREs in $X_{w}$. Thus, the decision of replenishment for a retailer considers its internal inventory cost increase as well as external CRE incentives from the supplier. The external 
incentives offered to the retailers by the supplier will be offset by the savings of the supplier's internal cost through the participations of the retailers in the MCRE. The supplier's savings results from tradeoffs between internal order processing costs for the retailers' joint orders through the MCRE and the retailers' original individual orders.

\subsection{Model analysis}

Before enacting an MCRE scheme, the optimal order interval of a retailer $i$ is $t_{i}=\sqrt{2 \mathrm{~K}_{i} / h_{i} \lambda_{i} P}$ with the inventory cost $g_{i}=\sqrt{2 \mathrm{~K}_{i} h_{i} \lambda_{i} P}$ and the total cost $T_{i}=P \lambda_{i}+g_{i}$. The supplier's cost includes an order and a delivery costs for each retailer, and then the total cost is given by $\sum_{i=1}^{m}\left(U_{i}+D_{i}\right) / t_{i}$. When an MCRE scheme is enacted, a retailer $i$ selecting a CRE $j$ will set its replenishment interval $t_{i j}=n_{i j} \tau_{j}$ to minimize its inventory cost under the CRE. Hence, the inventory cost of the retailer $i$ resulting from participating in the CRE $j$ is $g_{i j}=g_{i}\left(t_{i} / t_{i j}+t_{i j} / t_{i}\right) / 2$ with the total cost $T_{i j}=\left(1-d_{j}\right) P \lambda_{i}+g_{i j}$. The optimal $n_{i j}$, denoted as $n_{i j} *$, such that $g_{i j}\left(n_{i j} *\right)$ is minimized can be solved by using the inequality $n_{i j}^{*}\left(n_{i j}^{*}-1\right) \leq 2 \mathrm{~K}_{i} / h_{i} \lambda_{i} P \tau_{j}^{2} \leq n_{i j}^{*}\left(n_{i j}^{*}+1\right)$. Then, we have $n_{i j}^{*}=\left\lfloor\left(1+\sqrt{1+8 K_{i} / h_{i} \lambda_{i} P \tau_{j}^{2}}\right) / 2\right\rfloor$ where $\lfloor x\rfloor$ is the largest integer not larger than $x$. Nevertheless, if $g_{i j} / g_{i} \geq \beta_{i}$, the CRE is not eligible to the retailer due to the cost increase beyond its internal tolerance. Conversely, if a retailer $i$ agrees with a CRE $j$, the supplier will offer a price discount and an extra savings to satisfy the inequality $d_{j} \lambda_{i} P+\left(1-S_{j}\right) g_{i} \geq g_{i j}$. The least discount offered by a CRE $j$ acceptable to a retailer $i$ is given by $\rho_{i j}=\left\{g_{i j}-\left(1-S_{j}\right) g_{i}\right\} / \lambda_{i} P$; this representation is similar to the one in Mishra (2004).

To avoid pre-segmenting retailers into any CRE, cf. Mishra's SD model, two very useful indicators are introduced to conduct the following analysis toward our model. First, let $\gamma_{i j} \in\{0,1\}$ be the binary participation indicator for a retailer $i$ to select a CRE $j ; \gamma_{i j}=1$ indicates that the selection is true, $\gamma_{i j}=0$, otherwise. Note that the retailer $i$ must choose a CRE from $X_{w}$ or hold its own order interval; that is, the sum of decision variables $\gamma_{i j}$ over all $j$ complies with $\sum_{j=1}^{w+1} \gamma_{i j}=1$. The second indicator is the feasibility indicator $\alpha_{i j}=\left\lceil g_{i} / g_{i j}-1 / \beta_{i}\right\rceil$ for all $i, j$, where $\lceil\mathrm{x}\rceil$ is the smallest integer not smaller than $x ; \alpha_{i j}=1$ indicates that retailer $i$ is eligible to select a CRE $j$, i.e., $g_{i j} / g_{i}<\beta_{i}, \alpha_{i j}=0$, otherwise. Notice that $\alpha_{i, w+1}=1$ for all $i$ since any retailer is always feasible to hold its own order interval, i.e., $g_{i, w+1} \equiv g_{i}$. In addition, these two indicators jointly satisfy an equality $\sum_{j=1}^{w+1} \alpha_{i j} \gamma_{i j}=1$ for all the retailers.

\subsection{General discount (GD) MCRE model}

Now we are ready to define the first MCRE model in this paper, the GD MCRE Model. In GD model, every retailer is free to join a CRE as long as the minimum total cost of the supplier can 
be reached. The problem of the general discount (GD) MCRE model is to determine which member of $X_{w}$ with corresponding $\tau_{j}, d_{j}$ utilized by all the retailers to minimize the supplier's total cost and can be formulated as follows:

Minimize

$$
G_{0}=\sum_{j=1}^{w}\left[\sum_{i=1}^{m} \gamma_{i j} / m\right\rceil \frac{A_{S}+A_{j}}{\tau_{j}}+\sum_{j=1}^{w} \sum_{i=1}^{m} \gamma_{i j}\left(d_{j} \lambda_{i} P+\frac{D_{i}}{n_{i j} \tau_{j}}\right)+\sum_{i=1}^{m} \gamma_{i, w+1} \frac{U_{i}+D_{i}}{t_{i}}
$$

subject to

$$
\begin{aligned}
& d_{j} \geq \alpha_{i j} \gamma_{i j} \rho_{i j}, \text { for } i=1, \ldots, m \text { and } j=1, \ldots, w, \\
& \sum_{j=1}^{w+1} \alpha_{i j} \gamma_{i j}=1, g_{i, w+1}=g_{i}, \text { for } i=1, \ldots, m, \\
& \gamma_{i j} \in\{0,1\}, \text { for } i=1, \ldots, m \text { and } j=1, \ldots, w+1, \\
& \left\{\tau_{1}, \ldots, \tau_{w}\right\} \in X_{w}
\end{aligned}
$$

By utilizing (1), once the supplier decides which CREs are applied in $X_{w}$, the problem is simply to determine the optimal MCRE scheme i.e., to decide the values of $d_{j}$ and $\gamma_{i j}$. The term $\left\lceil\sum_{i=1}^{m} \gamma_{i j} / m\right\rceil$ in (1) is to detect whether or not a CRE $j$ is utilized by any retailers. Employing the participation indicators allows us to process all the $w$ CREs simultaneously. The constraints (2) (5) allow GD to achieve minimum total cost in (1) for all the retailers with their best choice of price discount under all the available CREs. Therefore, GD Model shall make a cost improvement over the SD model in Mishra (2004).

Piplani and Viswanathan (2004) state that the models of Viswanathan and Piplani (2001) and Mishra (2004) may overestimate the order processing cost per unit time of the supplier as more than one CRE order occurs at the same time. By assuming a linear relationship of two CREs, Piplani and Viswanathan offer a procedure with three factors to remove the problem of order processing cost redundancy in the numerical examples. However, the redundancy of order processing costs $G_{0}$ in (1) may hide the true optimal combination of the MCRE and discounts from the optimal solutions.

\subsection{Exact general discount (EGD) MCRE model}

In modern retailing industry, a supplier often utilizes a distribution center to process retailers' order, the cost including order-picking and consolidation processes should be considered as a whole as long as the processes occur within a certain time frame. As a consequence, the supplier's relevant order processing costs may occur cost redundancy in GD model. The second MCRE model defined in the study, EGD, is designed to remove redundancy of the joint order processing cost in the GD model with a broader definition, and shall outperform the other models to reach a better coordination. To alleviate the order processing cost redundancy of $G_{0}$ in (1), first of all, we develop an exact formula to calculate the numbers of orders or of CRE processed simultaneously at certain points of time. Thus, we are able to conclude how many orders and CRE are processed during a certain period of time. 
In a practical sense, each CRE can be represented by a rational number with a scale measured in days, weeks, months, years, etc.; that is, all the elements in $X$ are rational numbers. Based on the characteristics of rational numbers, there exists a largest real number $V$ for the set $X_{w}$ such that the ratio $\tau_{j} / V$ equals a positive integer $l_{j}$ for all $j$. All the orders released by a retailer $i$ selecting a CRE $j$ will be placed at time interval $n_{i j} \tau_{j}$, i.e., $n_{i j} l_{j} V$, in which $V$ can be interpreted as a unit length of all the CREs in $X_{w}$. Define an integer $L$ as the least common multiplier of $n_{i j} l_{j}$ for all $i, j$, and then the time epoch $L V$ is able to cover all possible retailers' orders based on any CRE in $X_{w}$ for at least one complete cycle. Furthermore, all the orders from the retailers joining any CRE in $X_{w}$ will be released at time $k V$, where $k=1, \ldots, L$. Observe that while the retailer $i$ is eligible to place an order at time $k V$, some of the time $k V$ can be reached by $n_{i j} \tau_{j}$ times a positive integer such that the value of $z_{i j k}=\left\lfloor k / n_{i j} j_{j}\right\rfloor-\left\lceil k / n_{i j} I_{j}\right\rceil$ either turns to 1 , or turns to zero. By considering with the participation indicator, $\gamma_{i j} z_{i j k}=1$ if and only if the retailer $i$ selects a CRE $j$ and places an order at time $k V$; otherwise, $\gamma_{i j} z_{i j k}=0$. Let $Z_{j k}=\sum_{i=1}^{m} \gamma_{i j} z_{i j k}$, which can be interpreted as the number of orders released at time $k V$ by all the retailers who select a CRE $j$. Further, define $Y_{j k}=\left\lceil Z_{j k} / m\right\rceil ; Y_{j k}=1$ be a sign of at least one order released at time $k V$ by all the retailers who join a $\operatorname{CRE} j$, and $Y_{j k}=0$, otherwise.

The roles of $Y_{j k}$ can be described by the following two dimensions. The first dimension is the summation of $Y_{j k}$ over the CRE $j, \sum_{j=1}^{w} Y_{j k}$, which represents how many CREs in $X_{w}$ having at least one retailers' order at time $k V$. Hence, $\left\lceil\sum_{j=1}^{w} Y_{j k} / w\right\rceil=1$ indicates that there is at least one CRE having orders at time $k V$, and $\left\lceil\sum_{j=1}^{w} Y_{j k} / w\right\rceil=0$, otherwise. Thus, define $\varphi=\sum_{k=1}^{L}\left[\sum_{j=1}^{w} Y_{j k} / w\right\rceil / L$ to represent the portion of $k V$, where $k=1, \ldots, L$, having at least one order from the retailers joining any CRE in $X_{w}$. As a result, for the time epoch $L V$, there are $L \varphi$ points of time having CRE orders with an order processing cost $A_{s}$ occurring at each time point. Hence, the total order processing cost for CRE orders during time epoch $L V$ is $L \varphi A_{s}$, and the total order processing cost per unit time is $\varphi A_{s} / \mathrm{V}$.

The second dimension describing the roles of $Y_{j k}$ is the summation of $Y_{j k}$ over the number $k_{1}$ $\sum_{k=1}^{L} Y_{j k}$, which represents how many points of time within time $L V$ having orders based on the CRE $j$. Thus, define $\varphi_{j}=\sum_{k=1}^{L} Y_{j k} / L$ to represent the portion of $k V$, where $k=1, \ldots, L$, having at least one order from the retailers joining the CRE $j$. As a result, for the time $L V$, there are $L \varphi_{j}$ points of time having orders from the CRE $j$ with an order processing cost $A_{j}$ 
occurring at each time point. Hence, the total order processing cost for CRE orders in $X_{w}$ during time $L V$ is $L \varphi_{j} A_{j}$, and the total order processing cost per unit time is $\varphi_{j} A_{j} / V$ for all $j$.

The supplier's total cost with savings of the overestimated order processing costs in (1) can be revised as follows:

$$
E G_{0}=\frac{\varphi A_{S}}{V}+\sum_{j=1}^{w} \frac{\varphi_{j} A_{j}}{V}+\sum_{j=1}^{w} \sum_{i=1}^{m} \gamma_{i j}\left(d_{j} \lambda_{i} P+\frac{D_{i}}{n_{i j} \tau_{j}}\right)+\sum_{i=1}^{m} \gamma_{i w+1} \frac{U_{i}+D_{i}}{t_{i}} .
$$

Equation (6) is the total cost function of the Exact General Discount (EGD) MCRE model. The definition of $Y_{j k}$, having orders through a particular CRE at a certain point of time, is to formulate $\varphi_{j}$ and $\varphi$ as the portion of ordering occasions having orders through a particular CRE and through any CRE in $X_{w}$, respectively. The explicit expressions of $\varphi_{j}$ and $\varphi$ in our model are in contrast to Piplani and Viswanathan (2004). They use three factors to reach the same effect of $\varphi$ but with numerical expressions by examples, and do not consider an ordering processing cost, such as $A_{j}$, for an individual order within a CRE. Consequently, by substituting (6) into (1), the EGD Model is definitely an improvement over the GD Model.

\section{Numerical examples}

Based on the development of our GD and EGD models, all the retailers have more freedom to choose from a set of replenishment epochs than the SD model as long as the minimum total cost of the supplier can be reached. Moreover, the cost redundancy for order processing is removed in EGD model to match up with the modern logistics operations in the retailing industry. The numerical examples developed in this section are to show the advantages of our GD and EGD models over Mishra's SD model. In addition, some managerial insights will also be drawn from the numerical examples. Thus, we employ the numerical examples used in Mishra (2004) with an identical set of fifteen retailers and various processing costs of the supplier. The numerical examples can be referred to how a set of retailers efficiently participating various subsets of distribution channels provided by the supplier with a set of replenishment epochs. The methodologies for the retailers to decide their own subsets are restricted by SD, GD, and EGD models. The demands with the costs of order setup and holding of fifteen retailers are shown in Table 1 . The value ranges of processing costs of the supplier are $A_{j}=0$, for all $j, U_{i}$ $=10,100,200,500,1000$ and $D_{i}=10,100,200,500,1000$, for all $i$, and $A_{s}=U_{i}$ or $3 U_{i}$. The supplier's preferable CRE set is $X$ (in weeks) $=\{1 / 7,3 / 7,1,2,3,4,5,6,7,8,9,10,10,11,12,13\}$. The saving percentage $S_{j}$ is assumed to be $10 \%$ for any CRE. In Section 4.1 , the numerical results of GD and Mishra's SD in a single CRE fashion will be shown. The numerical results between EGD and SD in a multiple CRE fashion will be compared in Section 4.2. 


\begin{tabular}{|c|c|c|c|c|c|c|c|c|c|c|c|c|c|c|c|}
\hline Retailer & 1 & 2 & 3 & 4 & 5 & 6 & 7 & 8 & 9 & 10 & 11 & 12 & 13 & 14 & 15 \\
\hline$K_{i}$ & 50 & 50 & 150 & 50 & 150 & 100 & 500 & 500 & 1500 & 500 & 1500 & 1000 & 5000 & 3000 & 3000 \\
\hline$\lambda_{i}$ (in mill.) & 2 & 1 & 2 & 0.5 & 1 & 0.5 & 2 & 1 & 2 & 0.5 & 1 & 0.5 & 2 & 1 & 0.5 \\
\hline$h_{i}$ & \multicolumn{15}{|c|}{0.15} \\
\hline
\end{tabular}

Table 1. Data for retailers

\subsection{CRE under general discount (GD) vs. selective discount (SD)}

This subsection describes single CRE models, in which the supplier offers a set of time epochs to all the retailers, and the retailers can only pick up a single time epoch. The objective of models is to minimize the supplier's relevant cost. We term our GD model using (1) with $\beta_{i}=2$ for all $i$ as GD0, and GD0 without retailers' choices as GD0N, i.e., $1 / \beta_{i}=0$, for all $i$. We first obtain the single CRE results of GDON and GDO, and then compare with the results of Mishra's SD model in Table 2. The first columns of SD, GDON and GDO in Table 2 indicate the savings (denoted as $\mathrm{S} \%$ and $\mathrm{D} \%$ denotes discount all through the paper) over the situation with no coordination. The second columns show the resulting CRE policies. The third column of GDON demonstrates the savings by excluding the retailers with retailers' choice, and the number of retailers excluded from the results of the second column. Table 2 shows that the savings of GDON are better than those of SD in 31 out of 50 examples, and the savings are the same for the remaining 19 examples. When considering the retailers' tolerance, 22 out 50 examples have at least one retailer over its tolerance with GDON, e.g., there are two retailers over their individual tolerances in Ex30. By excluding those retailers out of these examples, the savings drop a little bit, e.g., the savings of Ex30 decline from $36.4 \%$ to $28.3 \%$. Now, by using model GD0, we are able to increase the savings for all 22 examples, and 12 out of 22 examples even have better savings than SD, e.g., the savings of Ex30 with GD0 is $28.3 \%$, where that of SD is only $25.1 \%$.

The numerical results in this subsection show that by relaxing pre-segmentation of the retailers in the SD model, GDON offers more flexibility to each retailer to select a better CRE. On the other hand, it is reasonable to see that GDO may have less cost savings of the supplier than GDON since GDO offers retailers' considerations in their cost disadvantages. GD0 shall be considered as a different model from GDON; actually, GDO incorporates the magnitude of the cost disadvantage of the retailer joining a CRE. 


\begin{tabular}{|c|c|c|c|c|c|c|c|c|c|c|}
\hline \multirow[b]{2}{*}{$E x$} & \multirow[b]{2}{*}{$\boldsymbol{A}_{s}$} & \multirow[b]{2}{*}{$\boldsymbol{U}_{i}$} & \multirow[b]{2}{*}{$D_{i}$} & \multicolumn{2}{|r|}{ SD } & \multicolumn{3}{|c|}{ GDON } & \multicolumn{2}{|r|}{ GDO } \\
\hline & & & & $S(\%)$ & $\begin{array}{c}\text { CRE- } \\
\text { D(\%)- } \\
\text { \# Included }\end{array}$ & $S(\%)$ & $\begin{array}{l}\text { CRE-D(\%)- } \\
\text { \# Included }\end{array}$ & $\begin{array}{c}S(\%)-\# \\
\text { excluded from } \\
\text { previous } \\
\text { column }\end{array}$ & $\mathbf{S}(\%)$ & $\begin{array}{l}\text { CRE-D(\%)- } \\
\text { \# Included }\end{array}$ \\
\hline 1 & \multirow{5}{*}{10} & \multirow{5}{*}{10} & 10 & 0.0 & -- & 0.0 & -- & -- & -- & -- \\
\hline 2 & & & 100 & 0.8 & $2-0.07-3$ & 5.5 & $2-0.11-2$ & -- & -- & -- \\
\hline 3 & & & 200 & 6.2 & $2-0.11-5$ & 12.0 & $3-0.23-3$ & -- & -- & -- \\
\hline 4 & & & 500 & 15.4 & $3-0.23-10$ & 25.1 & $4-0.36-6$ & $17.3-1$ & 22.4 & $3-0.23-6$ \\
\hline 5 & & & 1000 & 25.2 & $3-0.23-10$ & 36.4 & $6-0.64-6$ & $17.2-2$ & 28.4 & 3-0.23-7 \\
\hline 6 & \multirow{5}{*}{100} & \multirow{5}{*}{100} & 10 & 31.7 & $2-0.11-6$ & 31.7 & $2-0.11-6$ & -- & -- & -- \\
\hline 7 & & & 100 & 23.8 & $2-0.11-6$ & 23.8 & $2-0.11-6$ & -- & -- & -- \\
\hline 8 & & & 200 & 20.9 & $2-0.11-5$ & 24.3 & $3-0.23-6$ & -- & -- & -- \\
\hline 9 & & & 500 & 25.6 & $3-0.23-10$ & 31.5 & $4-0.36-7$ & $22.1-1$ & 29.3 & $3-0.23-7$ \\
\hline 10 & & & 1000 & 30.0 & $3-0.23-10$ & 39.1 & $6-0.64-6$ & $18.6-2$ & 31.8 & $3-0.23-7$ \\
\hline 11 & \multirow{5}{*}{200} & \multirow{5}{*}{200} & 10 & 48.6 & $2-0.12-8$ & 48.6 & $2-0.12-8$ & -- & -- & -- \\
\hline 12 & & & 100 & 38.7 & $2-0.12-8$ & 38.7 & $2-0.12-8$ & -- & -- & -- \\
\hline 13 & & & 200 & 32.9 & $2-0.12-8$ & 36.0 & $3-0.23-9$ & -- & -- & -- \\
\hline 14 & & & 500 & 33.9 & $3-0.23-10$ & 37.1 & $4-0.36-7$ & $26.1-1$ & 35.5 & $3-0.23-8$ \\
\hline 15 & & & 1000 & 34.4 & $3-0.23-10$ & 41.6 & $6-0.64-6$ & $21.0-2$ & 35.2 & $3-0.23-8$ \\
\hline 16 & \multirow{5}{*}{500} & \multirow{5}{*}{500} & 10 & 63.3 & $2-0.17-10$ & 63.3 & $2-0.17-10$ & -- & -- & -- \\
\hline 17 & & & 100 & 57.7 & $3-0.23-10$ & 57.7 & $3-0.23-10$ & -- & -- & -- \\
\hline 18 & & & 200 & 54.5 & $3-0.23-10$ & 54.5 & $3-0.23-10$ & -- & -- & -- \\
\hline 19 & & & 500 & 48.9 & $3-0.26-12$ & 49.6 & $4-0.36-11$ & $37.0-1$ & 48.9 & $3-0.26-12$ \\
\hline 20 & & & 1000 & 45.4 & $4-0.36-14$ & 49.6 & $6-0.64-10$ & $26.7-2$ & 44.5 & $3-0.26-12$ \\
\hline 21 & \multirow{5}{*}{1000} & \multirow{5}{*}{1000} & 10 & 74.7 & $3-0.26-12$ & 74.7 & $3-0.26-12$ & -- & -- & -- \\
\hline 22 & & & 100 & 71.5 & $3-0.26-12$ & 71.5 & $3-0.26-12$ & -- & -- & -- \\
\hline 23 & & & 200 & 68.6 & $3-0.26-12$ & 68.6 & $3-0.26-12$ & -- & -- & -- \\
\hline 24 & & & 500 & 62.0 & $3-0.26-12$ & 62.6 & $4-0.36-12$ & 46.7-1 & 62.0 & $3-0.26-12$ \\
\hline 25 & & & 1000 & 58.0 & $5-0.50-15$ & 59.0 & $5-0.50-12$ & $43.9-1$ & 55.5 & $3-0.26-12$ \\
\hline 26 & \multirow{5}{*}{30} & \multirow{5}{*}{10} & 10 & 0.0 & -- & 0.0 & -- & -- & -- & -- \\
\hline 27 & & & 100 & 0.0 & -- & 3.7 & $2-0.11-2$ & -- & -- & -- \\
\hline 28 & & & 200 & 5.3 & $2-0.11-5$ & 11.4 & $3-0.23-3$ & -- & -- & -- \\
\hline 29 & & & 500 & 15.1 & $3-0.23-10$ & 24.9 & $4-0.36-6$ & $17.1-1$ & 22.2 & $3-0.23-6$ \\
\hline 30 & & & 1000 & 25.1 & $3-0.23-10$ & 36.4 & $6-0.64-6$ & $17.14-2$ & 28.3 & $3-0.23-7$ \\
\hline 31 & \multirow{5}{*}{300} & \multirow{5}{*}{100} & 10 & 14.2 & $2-0.11-6$ & 14.2 & $2-0.11-6$ & -- & -- & -- \\
\hline 32 & & & 100 & 14.2 & $2-0.11-6$ & 14.2 & $2-0.11-6$ & -- & -- & -- \\
\hline 33 & & & 200 & 14.5 & $2-0.11-5$ & 20.0 & $3-0.23-6$ & -- & -- & -- \\
\hline 34 & & & 500 & 23.4 & $3-0.23-10$ & 29.9 & $4-0.36-7$ & $20.45-1$ & 27.2 & $3-0.23-7$ \\
\hline 35 & & & 1000 & 28.8 & $3-0.23-10$ & 38.5 & $6-0.64-6$ & $18.00-2$ & 30.6 & $3-0.23-7$ \\
\hline 36 & \multirow{5}{*}{600} & & 10 & 30.2 & $2-0.12-8$ & 30.2 & $2-0.12-8$ & -- & -- & -- \\
\hline 37 & & & 100 & 25.8 & $2-0.12-8$ & 27.8 & $3-0.23-8$ & -- & -- & -- \\
\hline 38 & & 200 & 200 & 26.4 & $3-0.23-10$ & 29.6 & $3-0.23-8$ & -- & -- & -- \\
\hline 39 & & & 500 & 30.2 & $3-0.23-10$ & 34.3 & $4-0.36-7$ & $23.31-1$ & 31.8 & $3-0.23-8$ \\
\hline 40 & & & 1000 & 32.3 & $3-0.23-10$ & 40.5 & $6-0.64-6$ & $18.81-2$ & 33.0 & $3-0.23-8$ \\
\hline 41 & 1500 & 500 & 10 & 49.1 & $3-0.23-10$ & 49.1 & $3-0.23-10$ & -- & -- & -- \\
\hline 42 & & & 100 & 47.0 & $3-0.23-10$ & 47.0 & $3-0.23-10$ & -- & -- & -- \\
\hline
\end{tabular}




\begin{tabular}{|c|c|c|c|c|c|c|c|c|c|c|}
\hline \multirow[b]{2}{*}{ Ex } & \multirow[b]{2}{*}{$A_{s}$} & \multirow[b]{2}{*}{$\boldsymbol{U}_{i}$} & \multirow[b]{2}{*}{$D_{i}$} & \multicolumn{2}{|r|}{ SD } & \multicolumn{3}{|c|}{ GDON } & \multicolumn{2}{|r|}{ GDO } \\
\hline & & & & $S(\%)$ & $\begin{array}{c}\text { CRE- } \\
\text { D(\%)- } \\
\text { \# Included }\end{array}$ & $S(\%)$ & $\begin{array}{l}\text { CRE-D(\%)- } \\
\text { \# Included }\end{array}$ & $\begin{array}{l}\text { S(\%)-\# } \\
\text { excluded from } \\
\text { previous } \\
\text { column }\end{array}$ & $\mathbf{S}(\%)$ & $\begin{array}{l}\text { CRE-D }(\%)- \\
\text { \# Included }\end{array}$ \\
\hline 43 & & & 200 & 45.3 & $3-0.23-10$ & 45.3 & $3-0.23-10$ & -- & -- & -- \\
\hline 44 & & & 500 & 42.4 & $3-0.26-12$ & 44.8 & $4-0.36-11$ & $32.19-1$ & 42.4 & $3-0.26-12$ \\
\hline 45 & & & 1000 & 42.8 & $5-0.50-15$ & 47.5 & $6-0.64-10$ & $24.56-2$ & 40.3 & $3-0.26-12$ \\
\hline 46 & & & 10 & 62.0 & $3-0.26-12$ & 62.0 & $3-0.26-12$ & -- & -- & -- \\
\hline 47 & & & 100 & 59.9 & $3-0.26-12$ & 60.4 & $4-0.36-13$ & $44.39-1$ & 59.9 & $3-0.26-12$ \\
\hline 48 & 3000 & 1000 & 200 & 58.5 & $4-0.36-14$ & 59.1 & $4-0.36-13$ & $43.11-1$ & 57.9 & $3-0.26-12$ \\
\hline 49 & & & 500 & 55.6 & $4-0.36-14$ & 56.2 & $4-0.36-13$ & $40.27-1$ & 53.5 & $3-0.26-12$ \\
\hline 50 & & & 1000 & 54.2 & $5-0.50-15$ & 55.5 & $6-0.64-11$ & $29.65-2$ & 49.1 & $3-0.26-12$ \\
\hline
\end{tabular}

Table 2. Numerical examples with single CRE

\subsection{MCRE under general discount vs. exact general discount}

The supplier offers a set of time epochs to all the retailers as described in subsection 4.1. Similar to the condition that the supplier offers a set of time epochs to all the retailers, in this subsection, however, any retailer can pick up a time epoch for her own as long as the supplier's relevant cost is minimized. Considering the MCRE scheme, we present numerical examples for a two-CRE problem. The result of seven examples with Ex 3, 8, 13, and 16 19 are compared as displayed in Table 3. Results in Table 3 show that GDON has better savings than SD in 4 out of 7 examples and equal values for the rest of 3 examples. Moreover, by removing the redundancy in processing costs from GDON, consider (6) without retailers' choices as EGDRN. EGDRN has better savings than GDON and SD in all 7 examples. EGDRN, GDON and SD have impacts not only on the savings but also on the resulting CRE policies. Table 3 also indicates the values of $V, L, \varphi, \varphi_{1}$, and $\varphi_{2}$ of the corresponding CREs in the second column of EGDRN. The processing cost factor $\bullet$ for entire CRE orders plays an important role of further savings; in contrast, the processing cost factors $\varphi_{1}$, and $\varphi_{2}$ for each CRE orders do not affect the savings since we assume $A_{1}=A_{2}=0$ in all the examples.

\begin{tabular}{|c|c|c|c|c|c|c|c|c|c|c|c|}
\hline \multirow[b]{2}{*}{ Ex } & \multicolumn{2}{|r|}{ SD } & \multicolumn{2}{|r|}{ GDON } & \multicolumn{7}{|c|}{ EGDRN } \\
\hline & $\begin{array}{c}S \\
(\%)\end{array}$ & $\begin{array}{c}\text { CRE - D(\%)- } \\
\text { \# Included }\end{array}$ & $\begin{array}{c}S \\
(\%)\end{array}$ & $\begin{array}{l}\text { CRE - D(\%)- \# } \\
\text { Included }\end{array}$ & $\begin{array}{c}S \\
(\%)\end{array}$ & $\begin{array}{c}\text { CRE - D(\%)- } \\
\text { \# Included }\end{array}$ & $\varphi$ & $\varphi_{1}$ & $\varphi_{2}$ & $\mathbf{v}$ & $\mathbf{L}$ \\
\hline 3 & 6.2 & $2-0.11-5 ; 3-0.08-1$ & 14.3 & $2-0.11-1 ; 3-0.17-2$ & 14.6 & $2-0.11-2 ; 4-0.21-2$ & 1.00 & 1.00 & 0.50 & 2 & 840 \\
\hline 8 & 21.8 & $2-0.11-6 ; 4-0.14-3$ & 26.4 & $2-0.11-3 ; 4-0.21-3$ & 28.0 & $2-0.11-3 ; 4-0.21-3$ & 1.00 & 1.00 & 0.50 & 2 & 840 \\
\hline 13 & 33.4 & $2-0.11-5 ; 4-0.14-3$ & 36.9 & $3-0.23-6 ; 4-0.17-3$ & 37.9 & $2-0.12-3 ; 3-0.17-6$ & 0.67 & 0.50 & 0.33 & 1 & 2520 \\
\hline 16 & 65.1 & $2-0.12-8 ; 8-0.25-3$ & 65.1 & $2-0.12-8 ; 8-0.25-3$ & 67.5 & $2-0.12-8 ; 8-0.25-3$ & 1.00 & 1.00 & 0.25 & 2 & 840 \\
\hline 17 & 57.8 & $2-0.12-8 ; 8-0.25-3$ & 57.8 & $2-0.12-8 ; 8-0.25-3$ & 60.7 & $2-0.12-5 ; 4-0.25-6$ & 1.00 & 1.00 & 0.50 & 2 & 840 \\
\hline 18 & 54.6 & $3-0.23-10 ; 9-0.26-2$ & 54.6 & $3-0.23-10 ; 9-0.26-2$ & 56.5 & $2-0.12-4 ; 4-0.25-7$ & 1.00 & 1.00 & 0.50 & 2 & 840 \\
\hline 19 & 49.0 & $3-0.23-10 ; 9-0.26-2$ & 51.5 & $4-0.36-6 ; 5-0.28-5$ & 52.3 & $2-0.12-2 ; 4-0.36-9$ & 0.50 & 0.50 & 0.50 & 2 & 840 \\
\hline
\end{tabular}

Table 3. Numerical examples with two CREs 
Similar to the single-CRE problem with consideration for retailers' tolerance, we set $\beta_{i}$ at either 2 or 1.5 for all the retailers of the examples in Table 3. As the results summarized in Table 4 indicate Ex 13, 18 and 19 are the only 3 out of 7 examples affected by the given tolerance limits. Furthermore, the effects are limited on GDON model for all 3 examples and the effect on EGDRN model occurs only for Ex19. Table 4 indicates that the impact of $\beta_{i}$ is not only on the number of retailers joining CREs, but also on the choice of the CRE. For example, in CDON of Ex13 with $\beta_{i}=1.5$, only a single CRE of $\tau=3$ is selected even though there are two CREs available. The impact of the tolerance limit may reduce the savings; however, the number of retailers joining CREs may increase or decrease. For instance, for EGDRN of Ex19 without retailers' choices, there are 11 retailers to join CREs, with $\beta_{i}=2$, there are 12 retailers, and with $\beta_{i}=1.5$, there are 10 retailers. The savings of GDON and EGDRN with or without retailers' tolerances for Ex19 are all better than the savings of SD. This indicates that GDON and EGDRN under the restriction of retailers' choices in this example are able to achieve a better coordination than the SD.

\begin{tabular}{|c|c|c|c|c|c|c|c|c|c|c|}
\hline \multirow[b]{2}{*}{ Ex } & \multirow[b]{2}{*}{$\beta_{i}$} & \multicolumn{2}{|r|}{ GDON } & \multicolumn{7}{|c|}{ EGDRN } \\
\hline & & $\begin{array}{c}S \\
(\%)\end{array}$ & $\begin{array}{c}\text { CRE - D(\%)- } \\
\text { \# Included }\end{array}$ & $S(\%)$ & CRE - D(\%)- \# Included & $\varphi$ & $\varphi_{1}$ & $\varphi_{2}$ & $\mathbf{v}$ & $\mathbf{L}$ \\
\hline \multirow{2}{*}{13} & 2.0 & 36.9 & $3-0.23-6 ; 4-0.17-3$ & 37.9 & $2-0.12-3 ; 3-0.17-6$ & 0.67 & 0.50 & 0.33 & 1 & 2520 \\
\hline & 1.5 & 26.0 & $3-0.17-5 ; 4-0.14-3$ & 37.9 & $2-0.12-3 ; 3-0.17-6$ & 0.67 & 0.50 & 0.33 & 1 & 2520 \\
\hline \multirow{2}{*}{18} & 2.0 & 54.6 & $3-0.23-10 ; 9-0.26-2$ & 56.5 & $2-0.12-4 ; 4-0.25-7$ & 1.00 & 1.00 & 0.50 & 2 & 840 \\
\hline & 1.5 & 40.3 & $3-0.20-9$ & 56.5 & $2-0.12-4 ; 4-0.25-7$ & 1.00 & 1.00 & 0.50 & 2 & 840 \\
\hline \multirow{2}{*}{19} & 2.0 & 37.3 & $4-0.29-4 ; 5-0.27-5$ & 51.2 & $2-0.17-4 ; 4-0.29-8$ & 1.00 & 1.00 & 0.50 & 2 & 840 \\
\hline & 1.5 & 21.8 & $4-0.44-6 ; 5-0.28-6$ & 50.2 & $2-0.12-4 ; 4-0.25-6$ & 1.00 & 1.00 & 0.50 & 2 & 840 \\
\hline
\end{tabular}

Table 4. Numerical Examples with two CREs and retailers' choices

\section{Concluding remarks}

The contribution of this research is threefold. First, we propose a general MCRE model under the criterion of minimizing the supplier's total cost without pre-segmenting all or a portion of the retailers into either one of the MCREs or none. Thus, our model can attain better savings than the selective discount model, and reach a better coordination for the supplier. Secondly, our general discount MCRE model further considers retailers' choices based on the limits of tolerance for the retailers. This mechanism allows the system to prevent any retailer's cost increase beyond its internal limit. Referring to the objective function in (1) with constraints (2) (4), our model can be easily modified to deal with the case of no retailers' choices, by defining the thresholds $\beta_{i}$ to a very large value such that $\alpha_{i j}=1$ for all the retailers and CREs. In general, a higher tolerance will reach a higher savings. Third, by defining the unit length of time for all the CREs, our model is able to incorporate additional processing costs $A_{j}$ for single CRE sets with an exact measurement, and our objective function in (6) extends Piplani and Viswanathan's model into a general formulation to alleviate order processing costs for entire 
CRE orders of the supplier. Moreover, if $A_{S}$ can be redefined as disregarding whether a retailer joins the MCRE or not, this case can be accommodated by incorporating all $t_{j}$ with all $\tau_{j}$, and then determine joint $V$ and $L$. Notice that the value of $Z_{j k}$ represent the number of orders placed through a CRE $j$ at time $k V$. The order processing cost $A_{j}$ for the CRE $j$ can be further extended from a constant to $A_{s}\left(Z_{j k}\right)$ which is a function of the number of orders.

All in all, this research delivers an Exact General Discount (EGD) MCRE model for a supplier to coordinate the ordering and replenishment processes of a set of heterogeneous retailers. The EGD model considers not only the minimization of the supplier's cost, but also the retailers' choices as well. The cost minimization in EDG helps the supplier reach a lower cost level than most of the earlier works mentioned previously. The mechanism of incorporating the retailers' choices in EGD allows the model to have a better fit into the retail industry where suppliers in supply chains may not have exclusive power to control all their retailers. Nevertheless, our model still has certain limitations, such as constant demand rates and instant replenishment lead time. Future research may incorporate variations in demand or/and lead time.

\section{References}

7-Eleven, Inc. (2012). Hoover's Company Records, 41970. Retrieved Septempter 1, 2012 from http://search.proquest.com/docview/230557190?accountid=10534.

Alimentation Couche-Tard, Inc. (2012). Hoover's Company Records, 104187. Retrieved Septempter 1, 2012 from http://search.proquest.com/docview/230569770? accountid=10534.

Arshinder, Kandaa, A., \& Deshmukh, S.G. (2008). Supply chain coordination: perspectives, empirical studies and research directions. International Journal of Production Economics, 115(2), 316-335. http://dx.doi.org/10.1016/j.ijpe.2008.05.011

Chan, H.K. (2011). Supply chain Systems- recent trend in research and applications. IEEE System Journal, 5(1), 2-5. http://dx.doi.org/10.1109/JSYST.2010.2100191

Chan, H.K., \& Chan, F.T.S. (2010). A review of coordination studies in the context of supply chain dynamics. International Journal of Production Research, 48(10), 2793-2819. http://dx.doi.org/10.1080/00207540902791843

Chen, T., \& Chen, J. (2005). Optimizing supply chain collaboration based on joint replenishment and channel coordination. Transportation Research Part E: Logistics and Transportation Review, 41(4), 261-285. http://dx.doi.org/10.1016/j.tre.2004.06.003

FamilyMart Co., Ltd. (2010). Hoover's Company Records, 55997. Retrieved Septempter 1, 2012 from http://search.proquest.com/docview/230577586? Accountid=10534. 
Feng, Y., \& Viswanathan, S. (2007). Impact of demand uncertainty on coordinating supply chain inventories through common replenishment epochs. Journal of the Operational Research Society, 58(7), 964-971. http://dx.doi.org/10.1057/palgrave.jors.2602219

Fugate, B., Sahin, F., \& Mentzer, T.J. (2006). Supply chain management coordination mechanisms. Journal of Business Logistics, 27(2), 129-161. http://dx.doi.org/10.1002/j.21581592.2006.tb00220.x

Goyal, S.K., \& Gupta, Y.P. (1989). Integrated inventory models: the buyer vendor coordination. European Journal of Operational Research, 41(3), 261-269. http://dx.doi.org/10.1016/0377-2217(89)90247-6

Jorgensen, S., \& Zaccour, G. (2003). Channel coordination over time: Incentive equilibria and credibility. Journal of Economic Dynamics and Control, 27(5), 801-822. http://dx.doi.org/10.1016/S0165-1889(01)00072-0

Li, X., \& Wang, Q. (2007). Coordination mechanisms of supply chain systems. European Journal of Operational Research, 179(1), 1-16. http://dx.doi.org/10.1016/j.ejor.2006.06.023

Mishra, A.K. (2004). Selective discount for supplier-buyer coordination using common replenishment epochs. European Journal of Operational Research, 153(3), 751-756. http://dx.doi.org/10.1016/S0377-2217(02)00811-1

Piplani R., \& Viswanathan, S. (2004). Supply chain inventory co-ordination through multiple, common replenishment epochs and selective discount. International Journal of Logistics: Research and Applications, 7(2), 109-118. http://dx.doi.org/10.1080/13675560410001670224

Sarmah, S.P., Acharya, D., \& Goyal, S.K. (2006). Buyer vendor coordination models in supply chain management. European Journal of Operational Research, 175(1), 1-15. http://dx.doi.org/10.1016/j.ejor.2005.08.006

Sarmah, S.P., Acharya, D., \& Goyal, S.K. (2008). Coordination of a single-manufacturer/multibuyer supply chain with credit option. International Journal of Production Economics, 111(2), 676-685. http://dx.doi.org/10.1016/j.ijpe.2007.04.003

Tsay, A.A., Nahmias, S., \& Agrawal, N. (1999). Modeling supply chain contracts: a review. In Tayur, S., Ganeshan, R., \& Magazine, M. (Eds.). Quantitative models for supply chain management (pp. 299-336). The Netherlands: Kluwer Academic Publishers. http://dx.doi.org/10.1007/978-1-4615-4949-9_10

Viswanathan, S., \& Piplani, R. (2001). Coordinating supply chain inventories through common replenishment epochs. European Journal of Operational Research, 129(2), 277-286. http://dx.doi.org/10.1016/S0377-2217(00)00225-3 
Weng, Z.K. (1995). Channel coordination and quantity discounts. Management Science, 41(9), 1509-1523. http://dx.doi.org/10.1287/mnsc.41.9.1509

Wu, S-H., \& Hwang, J. (2011). Inventory policies for a supplier facing mixed periodic demand in a single-supplier multi-retailer supply chain. International Journal of Services and Operations Management, 9(1), 32-51. http://dx.doi.org/10.1504/IJSOM.2011.040320

Yao, M., \& Chiou, C. (2004). On a replenishment coordination model in an integrated supply chain with one vendor and multiple buyers. European Journal of Operational Research, 159(2), 406-419. http://dx.doi.org/10.1016/j.ejor.2003.08.024

Yao, M., \& Chiou, C. (2009). A new cooperative scenario for supply chains using common replenishment epochs. Journal of the Operations Research Society of Japan, 52(3), 263-282.

Journal of Industrial Engineering and Management, 2013 (www.jiem.org)

Article's contents are provided on a Attribution-Non Commercial 3.0 Creative commons license. Readers are allowed to copy, distribute and communicate article's contents, provided the author's and Journal of Industrial Engineering and Management's names are included. It must not be used for commercial purposes. To see the complete license contents, please visit http://creativecommons.org/licenses/by-nc/3.0/. 